\title{
A REMARK ON DERIVATIONS AND SKEW-DERIVATIONS ON $\mathscr{D}(M)$
}

\author{
TSUNEO SUGURI
}

\begin{abstract}
We give here the corrections of the Proposition of $\mathrm{S}$. Kobayashi and K. Nomizu concerning the derivation and skew-derivation of the algebra of differential forms on a differentiable manifold.
\end{abstract}

Let $M$ be an $n$-dimensional differentiable manifold, and $\mathscr{D}^{r}(M)$ the space of differential forms of degree $r$ defined on $M$. Then with respect to the exterior product, $\mathscr{D}(M)=\sum_{r=0}^{n} \mathscr{D}^{r}(M)$ forms an algebra over the real field R. A derivation or a skew-derivation of $\mathscr{D}(M)$ is a linear mapping of $\mathscr{D}(M)$ into $\mathcal{D}(M)$ satisfying the following condition:

(1) $D$ : derivation:

$$
D\left(\omega \wedge \omega^{\prime}\right)=D \omega \wedge \omega^{\prime}+\omega \wedge D \omega^{\prime}, \text { for } \omega, \omega^{\prime} \in \mathcal{D}(M) .
$$

(2) $D$ : skew-derivation:

$$
D\left(\omega \wedge \omega^{\prime}\right)=D \omega \wedge \omega^{\prime}+(-1)^{r} \omega \wedge D \omega^{\prime}, \quad \text { for } \omega \in \mathscr{D}^{r}(M), \omega^{\prime} \in \mathscr{D}(M) .
$$

A derivation or a skew-derivation $D$ of $\mathscr{D}(M)$ is said to be of degree $k$ if it maps $\mathscr{Q}^{r}(M)$ into $\mathscr{Q}^{r+k}(M)$ for every $r$. Then the following proposition is given in S. Kobayashi and K. Nomizu [2].

Proposition. (a) If $D$ and $D^{\prime}$ are derivations of degree $k$ and $k^{\prime}$, respectively, then $D D^{\prime}-D^{\prime} D$ is a derivation of degree $k+k^{\prime}$.

(b) If $D$ is a derivation of degree $k$ and $D^{\prime}$ is a skew-derivation of degree $k^{\prime}$, then $D D^{\prime}-D^{\prime} D$ is a skew-derivation of degree $k+k^{\prime}$.

(c) If $D$ and $D^{\prime}$ are skew-derivations of degree $k$ and $k^{\prime}$, respectively, then $D D^{\prime}+D^{\prime} D$ is a derivation of degree $k+k^{\prime}$.

(d) A derivation or a skew-derivation is completely determined by its effect on $\mathscr{D}^{0}(M)=\mathscr{F}(M)$ and $\mathscr{D}^{1}(M)$.

Recently, through discussions at the Research Institute of Mathematics, Tamkang College, I find that the conclusions (b) and (c) of the Proposition should be corrected as follows.

Proposition. [Case B]. If $D$ is a derivation of degree $k$ and $D^{\prime}$ is a skew-derivation of degree $k^{\prime}$, then we have:

(1) If $k$ is even, then $D D^{\prime}-D^{\prime} D$ is a skew-derivation of degree $k+k^{\prime}$.

(2) If $k$ is odd, then there exists no nonzero derivation nor skew-derivation of the type $D D^{\prime}-D^{\prime} D$ or of the type $D D^{\prime}+D^{\prime} D$.

[Case C]. If $D$ and $D^{\prime}$ are skew-derivations of degree $k$ and $k^{\prime}$, respectively, then we have:

Received by the editors November 12, 1974 and, in revised form, February 6, 1975.

AMS (MOS) subject classifications (1970). Primary 58A05, 58A10.

Key words and phrases. Algebra of differential forms, derivation, skew-derivation. 
(1) If $k$ and $k^{\prime}$ are both even, then $D D^{\prime}-D^{\prime} D$ is a derivation of degree $k+k^{\prime}$.

(2) If $k$ and $k^{\prime}$ are both odd, then $D D^{\prime}+D^{\prime} D$ is a derivation of degree $k+k^{\prime}$.

(3) If one of $k$ or $k^{\prime}$ is even and the other is odd, then there exists no nonzero derivation nor skew-derivation of the type $D D^{\prime}-D^{\prime} D$ or of the type $D D^{\prime}+$ $D^{\prime} D$.

The verifications of Cases $B$ and $C$ are straightforward, so we omit them. N. B. (i) See [1] for a systematic discussion of these questions.

(ii) F. W. Warner [3] calls a skew-derivation an antiderivation.

\section{REFERENCES}

1. A. Frölicher and A. Nijenhuis, Theory of vector-valued differential forms. I. Derivations in the graded ring of differential forms, Nederl. Akad. Wetensch. Proc. Ser. A $59=$ Indag. Math. 18(1956), 338-359. MR18, 569.

2. S. Kobayashi and K. Nomizu, Foundations of differential geometry. Vol. I, Wiley, New York, 1963. MR27 \#2945.

3. F. W. Warner, Foundations of differentiable manifolds and Lie groups, Scott, Foresman, Glenview, Ill., 1971. MR45 \#4312.

Department of Mathematics, Faculty of Science, Kyushu University, Fukuoka, 812 JAPAN (Current address)

Research Institute of Mathematics, Tamkang College of Arts and Sciences, Tamsui, TAIPEI, TAIWAN 